\title{
Stress begets stress: the association of adverse childhood experiences with psychological distress in the presence of adult life stress
}

\author{
Mercy Manyema ${ }^{1,2^{*}}$, Shane A. Norris ${ }^{2}$ and Linda M. Richter ${ }^{1}$
}

\begin{abstract}
Background: Adverse childhood experiences (ACES) have been linked to poor health and well-being outcomes, including poor mental health such as psychological distress. Both ACEs and psychological distress pose a significant public health burden, particularly in low to middle income countries. Contemporaneous stress events in adulthood may also impact psychological distress. The aims of this study were to describe the prevalence of ACEs and psychological distress and to assess the separate and cumulative effect of ACEs on psychological distress, while accounting for the effect of adult stress.

Methods: In this cross-sectional study, we used retrospectively measured ACEs from a sample of 1223 young adults aged between 22 and 23 years (52\% female) from the Birth to Twenty Plus Study. Psychological distress and adult life stress were measured with a six-month recall period. Hierarchical logistic regression was employed to assess the associations between the exposures and outcome.

Results: Nearly $90 \%$ of the sample reported at least one ACE and 28\% reported psychological distress. The median number of ACEs reported was three (range 0-11). After accounting for demographic and socio-economic factors, all ACEs were individually associated with psychological distress except for parental divorce and unemployment. The individual ACEs increased the odds of PD by between 1.42 and 2.79 times. Compared to participants experiencing no ACEs, those experiencing one to five ACEs were three times more likely to report psychological distress (AOR 3.2 95\% Cl: 1.83-5.63), while participants who experienced six or more ACEs had nearly eight times greater odds of reporting psychological distress (AOR 7.98 95\% Cl: 4.28-14.91). Interaction analysis showed that in the absence of adult life stress, the effect of low ACEs compared to high ACEs on PD was not significantly different.

Discussion and conclusion: The prevalence of ACEs in this young adult population is high, similar to other studies in young adult populations. A significant direct association exists between ACEs and psychological distress. Adult life stress seems to be a mediator of this relationship. Interventions targeted at psychological distress should address both early life adversity and contemporary stress.
\end{abstract}

Keywords: Adverse childhood experiences, Psychological distress, Mental health, Stressful life events, Young adult, Birth to twenty plus

\footnotetext{
* Correspondence: mercy.manyema@gmail.com

${ }^{1}$ DST-NRF Center of Excellence in Human Development, University of the Witwatersrand, 1st Floor School of Public Health Building, Wits Education Campus, 7 York Road, Parktown, Johannesburg 2193, South Africa

${ }^{2}$ MRC/Wits Developmental Pathways for Health Research Unit, Corner

College \& Clinic Road, Chris Hani Baragwanath Academic Hospital, University

of the Witwatersrand, Johannesburg, South Africa
}

(c) The Author(s). 2018 Open Access This article is distributed under the terms of the Creative Commons Attribution 4.0 International License (http://creativecommons.org/licenses/by/4.0/), which permits unrestricted use, distribution, and reproduction in any medium, provided you give appropriate credit to the original author(s) and the source, provide a link to the Creative Commons license, and indicate if changes were made. The Creative Commons Public Domain Dedication waiver (http://creativecommons.org/publicdomain/zero/1.0/) applies to the data made available in this article, unless otherwise stated. 


\section{Background}

Adverse childhood experiences (ACEs) have been linked to a myriad of poor behavioral and health outcomes. ACEs as defined by the Kaiser-Permanente Study, also known as the ACE Study, include child abuse and neglect and growing up in dysfunctional households characterized by domestic violence, mental illness and incarceration of a household member, parental divorce or separation and household drug or alcohol abuse [1]. An increasing body of evidence points to a consistent link between ACEs and poor mental health in both children and adults. The ACE Study itself showed that the risk of suicide attempts increased two to five-fold with experiencing any ACE and an ACE score of four or more was associated with increased risk of attempted suicide, lifetime depressive disorders and poor mental health in general [2-4]. Strong dose-response relationships between ACEs and depressive symptoms, drug abuse and antisocial behavior as well as psychological distress (PD), perceived well-being and missed days of work have also been demonstrated $[5,6]$. A South African study, though not using the full description of ACEs, found a significant association between childhood emotional neglect and adult depression, alcohol problems and suicidality [7].

A high proportion of children from different settings are exposed to ACEs. The ACE study reported a $60 \%$ prevalence of at least one ACE and 10\% five or more ACEs $[1,8]$. Nationally representative surveys conducted in England and Wales revealed that 46 and $47 \%$ of people, respectively, suffered at least one $\mathrm{ACE}$ and 8.3 and $14 \%$ respectively experienced four or more ACEs $[9,10]$. In Brazil, $85 \%$ of an adolescent cohort reported experiencing at least one ACE in their lifetime [11]. According to Jewkes et al., 54.7 and $56.4 \%$ of rural South African women and men experience emotional abuse respectively, 41.6 and $39.6 \%$ experienced emotional neglect, and 39.1 and $16.7 \%$ experienced sexual abuse before the age of 18 [7].

Mental, neurological and substance use disorders constitute a significant global disease burden, with $10 \%$ of global disability-adjusted life years (DALYs) attributed to these disorders in 2010 [12, 13]. More than 1.1 billion people worldwide suffered from mental and substance use disorders in 2016 according to the Global Burden of Disease Study, constituting $18.6 \%$ of the total non-fatal disease burden [14]. In the South African Stress and Health (SASH) Study conducted between 2002 and 2004, the lifetime prevalence of major depression was 9.7, and $4.9 \%$ for the past year; 9.8 and $4.9 \%$ for mood disorders, and 15.8 and $8.1 \%$ for anxiety disorders respectively $[15,16]$. Mental health remains neglected in many countries with a large gap existing between the burden caused and resources available to address mental health problems [17]. PD, which is defined as a "state of emotional suffering" characterized by depressive, anxiety as well as some somatic symptoms, is widely used as a marker of population mental health [18]. Evidence shows that it is positively associated with mental disorders and mental well-being [19].

The occurrence of stressful life events in adulthood increases the likelihood of experiencing psychological distress, as do race and availability of material resources, according to the SASH Study [20]. A complex interplay exists between early life stress, socioeconomic status and later life adversity. The stress sensitization theory has been posited as a possible mechanism through which early adversity and stress in later life and poor health are connected. It suggests that repeated stress early in life dysregulates stress response systems and lowers the threshold for reactivity and adaptive responses to subsequent stress. This increases the risk of mental health disorders in later life [16, 21]. In the same vein, Pearlin et al. put forward the theory of stress proliferation, a process through which stress begets stress, i.e. exposure to serious adversity in childhood increases the risk for later exposure to additional adversities [21, 22]. Childhood adversity can result in secondary stressors that cause harmful health consequences either together with or in place of the initial event, with some secondary stressors possibly being other adverse events [22].

Several other factors have been identified as correlates of poor mental health. The prevalence of major depression and PD has been found to be higher among females than males, among people with lower levels of education and among those aged 40 to 49 years [15, 23]. Increased risk of PD has been reported for unemployed and disabled persons as well as for those experiencing financial difficulties [23-25]. Some evidence suggests that household dysfunction ACEs are linked with the highest risk of mental disorders compared to abuse and neglect [26].

The relationship between ACEs and mental health is of marked public health importance. This association should however be examined in light of the several factors that may influence it, including adult stress and SES. The majority of literature on the epidemiology of ACEs and their impact on mental health come from high-income countries. The objectives of this study were: (1) to describe the prevalence of ACEs and PD in a young adult South African sample, (2) to examine the association between individual ACEs and PD and (3) to assess the cumulative effect of ACEs on PD, while accounting for the effect of adult stress on this relationship.

\section{Methods}

\section{Data and study population}

Data for this cross-sectional study were drawn from the Birth to Twenty Plus (Bt20+) Study. Named the Birth to Ten Study (Bt10) at its inception, the Bt20+ has been following up urban children and tracking their growth, 
health, well-being and educational progress from 1990 to date [27]. The study is based in Soweto, a densely populated suburb in the Greater Johannesburg Metropolitan area in South Africa, with an estimated population of about 1.5 million in 2017 [27, 28]. In the period between 23 April and 8 June 1990, 3273 singleton children born to women resident in Soweto-Johannesburg during the designated 7-week enrolment period and for a further 6 months post-partum were recruited into the study [27, 29]. More recruitment details are described elsewhere [27]. Of the initial cohort, 1636 were interviewed between 2012 and 2013 when the cohort was aged between 22 and 23 years and data for this analysis were drawn from this data collection wave.

\section{Measures}

\section{Psychological distress}

The main outcome measure, psychological distress (PD), was assessed using the General Health Questionnaire 28 (GHQ-28), a screening tool developed to test the risk of developing psychiatric disorders [30]. It comprises 28 items arranged in four subscales: somatic symptoms; anxiety/insomnia; social dysfunction, and severe depression. Each item has four possible responses namely Not at all, No more than usual, Rather more than usual, and Much more than usual, coded 0 to 3 . The recall period for the GHQ-28 is 6 months. The total score for each participant was computed with a possible score of 21 for each subscale and a global total of 84 . In line with literature, a total score of 24 was considered to be the threshold for PD, creating a binary variable for PD [30]. The four subscales were also analysed separately as count variables. The internal validity of the GHQ-28 was tested using Cronbach's alpha analysis. An alpha coefficient of 0.9013 was obtained for correlation between all the 28 items and values of $0.7649,0.8541,0.7608$ and 0.8802 were obtained for the somatic, anxiety, social dysfunction depression subscales respectively. All the questionnaire items were therefore retained in the analysis.

\section{Adverse childhood experiences}

Using a questionnaire adapted from the ACE Study Questionnaire [31], ACEs were retrospectively reported at 22-23 years of age. Data on chronic illness, unemployment and parental death were also collected in addition to the original ten ACEs used in the ACE study, based on recommendations to include some experiences that may be prevalent and relevant in low-income settings [32]. Additional File 1 shows the questions used to assess ACEs. A point was allocated to each ACE exposure for which a participant answered yes to one or both questions, i.e. only one point could be allocated to each ACE.
ACEs were analyzed as single variables, or grouped into abuse (emotional, physical and sexual abuse), neglect (emotional and physical neglect) and household dysfunction (parental divorce, parental death, drug abuse in household, mentally ill household member, chronically ill household member, incarcerated household member, witnessing domestic violence and unemployment of caregiver) categories. An ACE score was computed by adding the number of ACEs to which each person was exposed. Three categorical variables were created based on the ACE score:

1. A binary variable to indicate the proportion of participants who had experienced at least one ACE before the age of 18 years.

2. A variable with five ACE score categories to indicate those who experienced no ACEs, one, two, three and four or more ACEs, to allow comparability with similar studies [1].

3. A variable was created to broadly divide the ACE score into three categories: no ACEs (score of 0 ), lower level of ACEs (score 1 to 5) and higher level of ACEs (score 6 to 13).

These three variations of the ACE score were created to gain a better understanding of the cumulative effect of ACEs on mental well-being, as well as to have results comparable to similar studies.

\section{Stressful life events in adulthood}

Data were collected on the participants' experience of stressful life events during the preceding 6 months. Participants at age 22-23 years completed a questionnaire asking if the following had happened: injury of a household member due to violence, family member was a victim of crime, witnessing a violent crime, illness of a close family member, death of a close family member, living with someone with a serious disability in the family, drug or alcohol abuse in the household, fighting with or alienation from a close family member or neighbour, and incarceration of a family member. With each item assigned one point, a score of stressful life events experienced was computed and a categorical variable created with three categories: no experience (0), low levels (score of 1 to 3 ) and high levels of adult stress (score of 4 or more).

\section{Socioeconomic and demographic factors}

Previous work done on the Bt20+ data suggests that a wealth index is useful in adjusting regression models for SES [33]. At 22-23 years of age, participants were asked questions regarding ownership of thirteen household assets: television, car, washing machine, fridge, phone, radio, microwave, cell phone, DVD/ 
Video player, DSTV (satellite television), computer, internet and medical aid /medical insurance. We assessed SES using a summative wealth index based on this list of assets. SES was analysed as a continuous variable with scores ranging between 1 and 13. The scores were normally distributed with the mean very close in value to the median.

The questionnaire also collected data on gender, age, current marital status, graduation from high school or completing matric, and current employment status.

\section{Statistical analysis \\ Descriptive statistics}

Prevalence of ACEs and demographic characteristics were described by the presence or absence of $\mathrm{PD}$, and chi-square and analysis of variance (ANOVA) tests used to test for significant differences between them. The Kruskal-Wallis test was used to assess the difference in the reporting of subscale symptoms among those reporting at least one ACE compared to those with no ACE. The distribution of the ACEs grouped into abuse, neglect and household dysfunction is also described.

\section{Logistic regression: Effects of individual ACEs on PD}

Logistic regression was used to determine the unadjusted and adjusted association between ACEs and PD as follows:

a) The association between each individual ACE and PD was tested in univariate analysis as well as adjusted for demographic, SES and adult stress factors

b) A model was created that included all individual ACE variables and the demographic factors to assess for overlapping effects between the ACES.

c) Negative binomial regression was used to test for associations between ACEs and the different GHQ subscales and adult life stress.

d) The association between ACEs and adult life stress were also tested in univariate and multivariate analysis

\section{Hierarchical logistic regression}

a) We used hierarchical regression to test the association between the cumulative ACE score variables and $\mathrm{PD}$ in order to assess the direct relationship, the potential multiple pathways linking them as well the cumulative effects of not only ACEs but also adult stressful life events. In step 1, we adjusted for demographic variables of age, gender and marital status. The second step added household SES, current employment and completion of high school. The adult life stress variable was added in the third step of the hierarchical regression.

b) An interaction term between ACEs and adult life stress was also tested in separate models, the term being added to the model in the third step. Figure 1 shows the conceptual framework used in the hierarchical analysis.

The framework hypothesizes a direct association between ACEs and PD independent of other factors. However, ACEs may act through SES and adult stress to impact mental well-being. SES factors themselves may also have an effect on adult stress. Demographic factors, not shown in the framework are potential confounders that may be associated with both ACEs and PD.

\section{Missing data}

We performed descriptive statistics on participants with missing ACE and PD data to check if there were any significant differences in demographic characteristics.

\section{Results}

Descriptive analysis

Table 1 presents the demographic characteristics of the sample and the prevalence of ACEs and PD.

The sample comprised 1223 participants with complete ACE data of whom $51 \%$ were women and $49 \%$ men. Nearly $90 \%$ of the sample reported experiencing at least

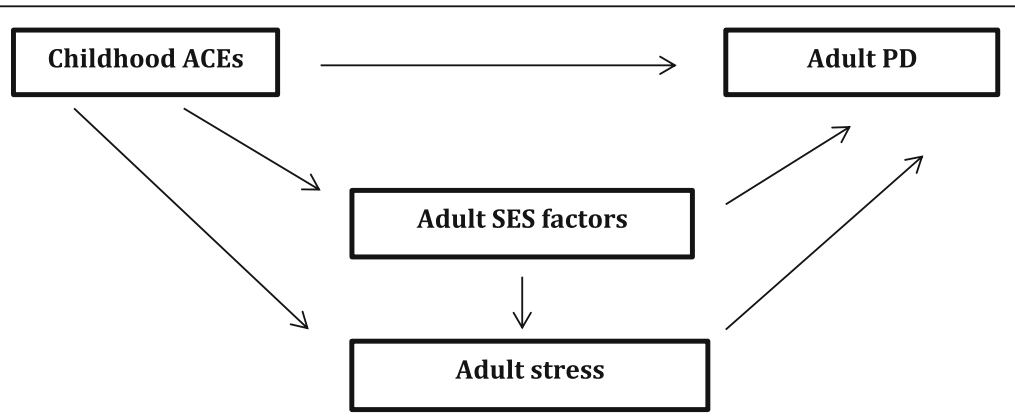

Fig. 1 Conceptual framework for the hierarchical regression. ACEs - Adverse childhood events; PD-psychological distress; SES-socioeconomic status 
Table 1 Prevalence of ACEs and demographic characteristics of the sample

\begin{tabular}{lllll}
\hline Variable & PD No n (\%) & PD Yes n (\%) & Total N (\%) & $p$-value \\
\hline Cumulative ACE Variables & &
\end{tabular}

Any ACE

$$
\text { No }
$$

Yes

$$
131(15)
$$$$
16(5)
$$$$
735(85)
$$$$
308(95)
$$

ACE score categories

$$
1 \text { ACE }
$$

1 ACE

2 ACES

3 ACES

4 or more ACES

Level of ACEs

$$
\begin{aligned}
& \text { None (0 ACEs) } \\
& \text { Low (1 to 5) }
\end{aligned}
$$

High (6 or more)

Abuse

$$
\text { No }
$$

Neglect

No
Yes

Household dysfunction

No
Yes

Yes

$\begin{array}{ll}169(16) & 29(7) \\ 892(84) & 400(93)\end{array}$

Emotional abuse

$\begin{array}{lll}\text { No } & 812(74) & 256(59) \\ \text { Yes } & 282(26) & 175(41)\end{array}$

Sexual abuse

$\begin{array}{lll}\text { No } & 1032(97) & 385(93) \\ \text { Yes } & 31(3) & 27(7)\end{array}$

Physical abuse

$$
\begin{aligned}
& \text { No } \\
& \text { Yes }
\end{aligned}
$$

\begin{tabular}{|c|c|c|c|c|}
\hline Variable & PD No n (\%) & PD Yes n (\%) & Total N (\%) & $\overline{p \text {-value }}$ \\
\hline \multicolumn{5}{|l|}{ Parental death } \\
\hline No & $794(73)$ & $305(70)$ & $1099(72)$ & \\
\hline Yes & $301(27)$ & $128(30)$ & $429(28)$ & 0.417 \\
\hline \multicolumn{5}{|l|}{ Substance abuse } \\
\hline No & $836(76)$ & $278(64)$ & $1114(73)$ & \\
\hline Yes & $264(24)$ & $155(36)$ & $419(27)$ & $<0.001$ \\
\hline \multicolumn{5}{|l|}{ Mental illness } \\
\hline No & $1003(91)$ & $345(80)$ & $1348(88)$ & \\
\hline Yes & $96(9)$ & $88(20)$ & $184(12)$ & $<0.001$ \\
\hline \multicolumn{5}{|l|}{ Imprisonment } \\
\hline No & $852(78)$ & $325(75)$ & $1177(77)$ & \\
\hline Yes & $246(22)$ & $108(25)$ & $354(23)$ & 0.289 \\
\hline \multicolumn{5}{|l|}{ Chronic illness } \\
\hline No & $837(76)$ & $292(68)$ & $1129(74)$ & \\
\hline Yes & $259(24)$ & $140(32)$ & $399(26)$ & $<0.001$ \\
\hline \multicolumn{5}{|l|}{ Unemployment } \\
\hline No & $644(59)$ & $222(51)$ & $866(57)$ & \\
\hline Yes & $456(41)$ & $211(49)$ & $667(43)$ & 0.010 \\
\hline Age & 23 (SD 0.6) & 23 (SD 0.6) & 23 (SD 0.6) & 0.494 \\
\hline \multicolumn{5}{|l|}{ Marital status } \\
\hline Single & $538(49)$ & $228(52)$ & $766(50)$ & \\
\hline Marital relationship & $567(51)$ & $207(48)$ & $774(50)$ & 0.188 \\
\hline \multicolumn{5}{|l|}{ Gender } \\
\hline Male & $594(54)$ & $150(34)$ & $744(48)$ & \\
\hline Female & $516(46)$ & $288(66)$ & $804(52)$ & $<0.001$ \\
\hline SES & 9.4 (SD 2.3) & 8.9 (SD 2.3) & 9.3 (SD 2.2) & 0.002 \\
\hline \multicolumn{5}{|c|}{ Secondary school graduation } \\
\hline No & $414(38)$ & $185(43)$ & $599(39)$ & \\
\hline Yes & $680(62)$ & $247(57)$ & $927(61)$ & 0.073 \\
\hline \multicolumn{5}{|l|}{ Currently employed } \\
\hline No & $450(47)$ & $219(57)$ & $669(50)$ & \\
\hline Yes & $503(53)$ & $166(43)$ & $669(50)$ & 0.001 \\
\hline \multicolumn{5}{|c|}{ Stressful life events (previous 6 months) } \\
\hline No & $352(33)$ & $85(20)$ & $437(29)$ & \\
\hline Low & $619(58)$ & $254(61)$ & $873(59)$ & \\
\hline High & $99(9)$ & 77 (19) & $176(12)$ & $<0.001$ \\
\hline
\end{tabular}

Emotional neglect

$\begin{array}{lll}\text { No } & 861(78) & 235(54) \\ \text { Yes } & 236(22) & 197(46)\end{array}$

Physical neglect

$\begin{array}{lllll}\text { No } & 992(91) & 355(82) & 1347(88) & \\ \text { Yes } & 101(9) & 77(18) & 178(12) & <0.001 \\ \text { Domestic violence } & & & & \\ \text { No } & 977(90) & 346(80) & 1323(87) & \\ \text { Yes } & 114(10) & 84(20) & 198(13) & <0.001\end{array}$

Parental divorce/separation

$\begin{array}{lllll}\text { No } & 534(57) & 179(51) & 713(55) & \\ \text { Yes } & 401(43) & 174(49) & 575(45) & 0.039\end{array}$

Table 1 Prevalence of ACEs and demographic characteristics of the sample (Continued)

one ACE, 35\% experienced at least four and 15\% experienced at least six ACEs. The most frequently reported ACEs were parental divorce or separation and parental unemployment ( 45 and $43 \%$ respectively), while the least frequently reported were sexual abuse (4\%) and physical abuse $(8 \%)$. Over a third of the participants reported experiencing some form of abuse, 33\% reported experiencing physical or emotional neglect and over $90 \%$ reported 
household dysfunction. The proportion of participants with PD was $28 \%$, with $66 \%$ of these being women. Approximately $50 \%$ of those who had PD reported experiencing at least four ACEs, compared to $30 \%$ of those who had no PD, and $25 \%$ reported six or more ACEs, compared to $11 \%$ of those who did not have PD. Of the demographic factors, there was no significant difference in education and marital status between those who reported PD and those who did not.

\section{Missing data}

Nearly $2 \%$ of males and $1.3 \%$ of females did not have complete data for both ACEs and PD. Twenty-one percent of males and $23 \%$ of the females did not have complete ACE data, while 1.6 and $2.4 \%$ did not have complete PD data respectively and were thus (by default) not included in the regression models.

\section{Reporting of individual GHQ subscales}

Presented in Additional File 2 are histograms that show the number of participants reporting each of the four different GHQ subscales in relation to their experience of at least one ACE. The median scores for the somatic, anxiety/insomnia, dysfunction and depression subscales were 4 (range 0-19), 4 (range 0-21), 6 (range 0-21) and 0 (range 0-21) respectively. The median scores for the subscales for participants experiencing at least one ACE compared to no ACEs were: 4 and 2 for somatic, 4 and 2 for anxiety/insomnia, 6 and 5.5 for dysfunction and 1 and 0 for depression respectively. The Kruskal-Wallis analysis showed that there was a significant difference in reporting somatic, anxiety/insomnia and depression symptoms between those who experienced at least one ACE and those who experienced no ACEs ( $p$-value $<0.05$ for all three).

\section{Regression analyses}

\section{Effects of ACEs on adult life stress}

Additional File 3 shows the separate and cumulative effects of ACEs on adult life stress. Compared to those who experienced no ACEs and low levels of ACEs, participants who reported high ACE levels had nearly twelve times greater odds of experiencing high levels of adult stress. All the demographic factors were not significantly associated with the experience of adult life stress. Parental death and sexual abuse were not significantly associated with adult life stress both in unadjusted and adjusted models.

\section{Separate effects of ACEs on PD}

The separate effects of ACEs on PD are presented in Table 2. Parental death and incarceration of household member were not associated with PD. After adjusting for demographic factors, parental divorce/separation and parental unemployment become statistically non-significant, pointing to possible confounding and/or mediation. In the model including all individual ACEs, the neglect ACEs and living with mental illness in the household remained statistically significantly increased the odds of PD, together with marital status, gender and adult life stress. Gender remained associated with PD in all adjusted models, with no evidence of reduction of effect size.

\section{Association between ACEs and the separate PD subscales}

The associations between the individual subscales and PD are shown in Additional File 4. The social dysfunction subscale was not statistically significantly associated with any of the cumulative ACE score variables, except experiencing at least six ACEs which was significant only in the univariate analysis. Of the demographic variables, household stress and gender significantly increased the relative risk of a high score in all four subscales. The depression subscale generally showed greater effect sizes compared to the other three subscales. A dose-response relationship is apparent in the three subscales that achieved statistical significance.

\section{Hierarchical logistic regression}

Table 3 shows the adjusted hierarchical regression analyses for the relationship between ACEs and PD, with the ACE score variable depicting three levels of ACEs: no ACEs, low level of ACES (1 to 5) and high level of ACEs (6 or more). Additional File 5 presents the same analyses using the binary ACE score variable.

The full regression models for the two ACE score exposures achieved significance. The likelihood ratio chi-square statistic showed statistically significant improvement between the null model and tested model after each step in the hierarchical modelling. The effect of ACEs remained statistically significant even after adjusting for demographic, SES and adult life events stress factors. A dose-response association is noted between the ACE score and PD. The addition of the demographic and SES factors increased the effect size of the ACE score to levels higher than the unadjusted values, but adult stress brought the ORs down in all the three full models to levels lower than or similar to unadjusted. Being married was not associated with PD in the unadjusted model but was significant when entered into the model with gender. The effect size of gender on PD increased at each subsequent level of the model.

In the models with the interaction terms, it is apparent that high levels of adult life stress increase the likelihood of PD by over 20 times compared to no stress, in the absence of ACEs, while low levels of stress have a statistically non-significant association. Having experienced at least one ACE was significantly associated with PD even in the absence of adult life event stress (AOR 2.74, 95\% 
Table 2 Effects of individual ACEs on PD

\begin{tabular}{|c|c|c|c|}
\hline Variable & Crude OR (95\% Cl) & Adjusted OR (95\% Cl) Individual ACEs ${ }^{1}$ & Adjusted OR (95\% Cl) All individual ACEs in combined model ${ }^{2}$ \\
\hline \multicolumn{4}{|c|}{ Emotional abuse } \\
\hline No & Ref & Ref & Ref \\
\hline Yes & $1.96(1.56-2.49)$ & $1.93(1.46-2.55)$ & $1.10(0.75-1.63)$ \\
\hline \multicolumn{4}{|l|}{ Sexual abuse } \\
\hline No & Ref & Ref & Ref \\
\hline Yes & $2.33(1.38-3.96)$ & $2.26(1.20-4.25)$ & $1.78(0.86-3.66)$ \\
\hline \multicolumn{4}{|l|}{ Physical abuse } \\
\hline No & Ref & Ref & Ref \\
\hline Yes & $1.75(1.17-2.60)$ & $1.87(1.20-2.93)$ & $1.14(0.67-1.94)$ \\
\hline \multicolumn{4}{|c|}{ Emotional neglect } \\
\hline No & Ref & Ref & Ref \\
\hline Yes & $3.05(2.41-3.88)$ & $2.79(2.10-3.70)$ & $1.99(1.34-2.93)$ \\
\hline \multicolumn{4}{|l|}{ Physical neglect } \\
\hline No & Ref & Ref & Ref \\
\hline Yes & $2.13(1.55-2.93)$ & $2.24(1.52-3.31)$ & $1.64(1.00-2.69)$ \\
\hline \multicolumn{4}{|c|}{ Domestic violence } \\
\hline No & Ref & Ref & Ref \\
\hline Yes & $2.08(1.53-2.83)$ & $1.91(1.35-2.72)$ & $1.22(0.78-1.90)$ \\
\hline \multicolumn{4}{|c|}{ Parental divorce/separation } \\
\hline No & Ref & Ref & Ref \\
\hline Yes & $1.29(1.01-1.65)$ & $1.13(0.85-1.51)$ & $0.96(0.70-1.31)$ \\
\hline \multicolumn{4}{|l|}{ Parental death } \\
\hline No & Ref & Ref & Ref \\
\hline Yes & $1.11(0.87-1.41)$ & $1.26(0.95-1.67)$ & $1.15(0.81-1.62)$ \\
\hline \multicolumn{4}{|c|}{ Substance abuse } \\
\hline No & Ref & Ref & Ref \\
\hline Yes & $1.77(1.39-2.25)$ & $1.63(1.22-2.17)$ & $1.24(0.86-1.80)$ \\
\hline \multicolumn{4}{|l|}{ Mental illness } \\
\hline No & Ref & Ref & Ref \\
\hline Yes & $2.66(1.95-3.65)$ & $2.72(1.89-3.91)$ & $2.25(1.45-3.51)$ \\
\hline \multicolumn{4}{|l|}{ Incarceration } \\
\hline No & Ref & Ref & Ref \\
\hline Yes & $1.15(0.89-1.98)$ & $1.12(0.83-1.52)$ & $0.79(0.54-1.16)$ \\
\hline \multicolumn{4}{|l|}{ Chronic illness } \\
\hline No & Ref & Ref & Ref \\
\hline Yes & $1.55(1.21-1.98)$ & $1.42(1.07-1.89)$ & $1.07(0.75-1.52)$ \\
\hline \multicolumn{4}{|l|}{ Unemployment } \\
\hline No & Ref & Ref & Ref \\
\hline Yes & $1.34(1.07-1.68)$ & $1.16(0.89-1.51)$ & $0.82(0.59-1.15)$ \\
\hline \multicolumn{4}{|l|}{ Marital status } \\
\hline Single & Ref & & Ref \\
\hline Relationship & $0.86(0.69-1.08)$ & & $0.64(0.50-0.87)$ \\
\hline \multicolumn{4}{|l|}{ Gender } \\
\hline Male & Ref & & Ref \\
\hline
\end{tabular}


Table 2 Effects of individual ACEs on PD (Continued)

\begin{tabular}{|c|c|c|c|}
\hline Variable & Crude OR (95\% Cl) & Adjusted OR (95\% CI) Individual ACEs ${ }^{1}$ & Adjusted OR (95\% Cl) All individual ACEs in combined model ${ }^{2}$ \\
\hline Female & $2.21(1.76-2.78)$ & & $2.73(1.98-3.77)$ \\
\hline SES & $0.91(0.87-0.96)$ & & $0.90(0.84-0.97)$ \\
\hline \multicolumn{4}{|c|}{ Completed matric } \\
\hline No & Ref & & Ref \\
\hline Yes & $0.81(0.65-1.02)$ & & $0.84(0.60-1.18)$ \\
\hline \multicolumn{4}{|c|}{ Currently employed } \\
\hline No & Ref & & Ref \\
\hline Yes & $0.68(0.53-0.86)$ & & $0.79(0.58-1.08)$ \\
\hline \multicolumn{4}{|c|}{ Stressful events } \\
\hline No & Ref & & Ref \\
\hline Low & $1.70(1.28-2.24)$ & & $1.67(1.14-2.45)$ \\
\hline High & $3.22(2.20-4.71)$ & & $1.80(1.05-3.08)$ \\
\hline
\end{tabular}

In unadjusted and adjusted analysis: bold $=p<0.05 ;{ }^{1}$ Each individual ACE entered as an exposure adjusted for demographic factors; all demographic factors except completing high school were significant at $p<0.05 ;{ }^{2}$ All individual ACEs placed in one multivariate model, also adjusted for demographic factors

CI 1.03-7.36). When the three category ACE score variable was used as the main exposure in Table 3, high levels of ACEs in the absence of adult stress increased the odds of PD by nearly 8 times. A closer look at the interaction terms shows that there was no significant difference in the effect of the level of ACEs on PD in the presence of low stress, i.e. the impact of one to five ACEs as well as experiencing six or more ACEs on PD did not differ significantly between those with low stress compared to those with no adult stress. However, both low and high levels of ACEs had a significantly different effect in individuals with high adult stress compared to those with no adult stress.

\section{Discussion}

In this sample of young South African adults, 87\% reported at least one ACE and 35\% reported four or more ACEs. Nearly a third of the population reported signs of PD, of which 66\% were women. Individual ACEs increased the odds of reporting PD by between 1.42 (chronic illness) and 2.79 (emotional neglect) times, after adjusting for demographic variables. Including all the ACEs in one model attenuated the effect of most ACEs except emotional and physical neglect and mental illness in the household. A significant direct association was observed between ACEs and PD, and a dose-response effect was apparent. A significant dose-response association was observed between ACE levels and adult stress levels with participants who reported experiencing six or more ACEs being 11 times more likely to experience high levels of adult stress (AOR 11.22; 95\% CI 7.1-17.8) compared to those who reported zero and one to five ACEs.

Our ACE prevalence levels are similar to those of the Kaiser-Permanente ACE study where over $60 \%$ of the population had experienced at least one ACE [1]. However they are more consistent with a later study that used an expanded version of the ACE list and found that over $80 \%$ of the participants reported at least one ACE [34]. Although the additional ACEs included in our analysis: parental death, parental unemployment and chronic illness in the household were among the most prevalent, their effect became non-significant in the model combining all individual ACEs. This may mean that their effect is manifest through other ACEs or that they act as moderating factors. As ACE research is widely used to advocate for the protection of and greater investment in the early childhood years, it is important for the ACE indices to be broadly focused in order to capture a diversity of experiences that may impact life-long health and wellbeing [32, 34].

The higher prevalence of PD among women compared to men is consistent with results from others studies [16, 35], although some studies have found no gender differentials in the prevalence of poor mental health [6]. Compared to men, women who experienced at least one ACE were two and half times more likely to report PD, an effect that increased with the inclusion of SES and adult stress in the model. Greater odds of women having PD given the experience of ACEs compared to men have been reported in other studies, including in South Africa [36, 37].

The effect of most single ACEs on PD attenuated and became statistically not significant after accounting for other ACEs and demographic and SES factors. These results imply that the effect of some ACEs may only manifest through other exposures and that ACEs often co-occur and are highly interrelated. In this sample, physical and emotional neglect and mental illness in the household seem to be the most foreboding individual ACE exposures impacting mental well-being independently of other ACEs, adult stress, SES and demographic factors. A dose-response effect was observed between the 
Table 3 Hierarchical regression results: ACEs as a three-category exposure

\begin{tabular}{|c|c|c|c|c|}
\hline & Model 1 & Model 2 & Model 3 & Model 4 (with interaction) \\
\hline $\mathrm{Chi}^{2} \Delta$ & $103.3^{\mathrm{a}}$ & 110.0 & 123.3 & 129.6 \\
\hline \multicolumn{5}{|l|}{ Level of ACEs } \\
\hline None (0 ACEs) & Ref & Ref & Ref & Ref \\
\hline Low (1 to 5) & $3.21(1.83-5.63)$ & $3.48(1.81-6.67)$ & $2.97(1.53-5.72)$ & $2.49(0.92-6.72)$ \\
\hline High (6 or more) & $7.98(4.28-14.91)$ & $8.57(4.19-17.53)$ & $6.54(3.14-13.63)$ & $7.67(1.90-30.94)$ \\
\hline \multicolumn{5}{|l|}{ Level of ACEs } \\
\hline No stressful life events\#No ACEs & & & & Ref \\
\hline Low stress\#LOW ACES & & & & $2.02(0.47-8.59)$ \\
\hline Low stress\#High ACEs & & & & $1.33(0.22-7.95)$ \\
\hline High stress\#Low ACEs & & & & $0.10(0.009-0.98)$ \\
\hline High stress\#High ACEs & & & & $0.08(0.006-0.95)$ \\
\hline Age & $1.10(0.85-1.40)$ & $1.09(0.84-1.44)$ & $1.09(0.84-1.45)$ & $1.09(0.83-1.44)$ \\
\hline \multicolumn{5}{|l|}{ Gender } \\
\hline Male & Ref & Ref & Ref & Ref \\
\hline Female & $2.61(1.97-3.45)$ & $2.67(1.97-3.63)$ & $2.71(2.00-3.71)$ & $2.77(2.03-3.79)$ \\
\hline \multicolumn{5}{|l|}{ Marital status } \\
\hline Single & Ref & Ref & Ref & Ref \\
\hline Relationship & $0.70(0.53-0.93)$ & $0.68(0.50-0.92)$ & $0.67(0.49-0.92)$ & $0.66(0.49-0.91)$ \\
\hline SES & & $0.91(0.85-0.98)$ & $0.91(0.84-0.98)$ & $0.91(0.84-0.98)$ \\
\hline \multicolumn{5}{|l|}{ Completed matric } \\
\hline No & & Ref & Ref & Ref \\
\hline Yes & & $0.79(0.57-1.09)$ & $0.81(0.58-1.12)$ & $0.81(0.58-1.13)$ \\
\hline \multicolumn{5}{|l|}{ Employment } \\
\hline No & & Ref & Ref & Ref \\
\hline Yes & & $0.79(0.59-1.07)$ & $0.80(0.59-1.09)$ & $0.80(0.59-1.08)$ \\
\hline \multicolumn{5}{|l|}{ Adult stress } \\
\hline None & & & Ref & Ref \\
\hline Low & & & $1.84(1.26-2.68)$ & $0.99(0.25-3.98)$ \\
\hline High & & & $2.25(1.35-3.74)$ & $21.92(2.35-204.86)$ \\
\hline
\end{tabular}

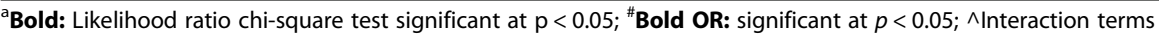

ACE score and PD, even after accounting for adult stress, as well as demographic and SES factors. This shows an unmediated, direct effect of ACEs on PD, and that this effect increases with exposure to a greater number of ACEs. This finding is in line with a growing body of evidence that shows that early life adversity can disrupt not only brain structure and functioning, but also dysregulate other systems resulting in low stress thresholds that persist throughout life and increase the risk of stress-related disease or disorders [38, 39]. This direct effect is however not the only effect of ACEs on PD. The hierarchical modelling showed increasing effects of ACEs with the addition of demographic and SES factors to the models. This may be due to exacerbating moderation effects of these factors on the association between ACEs and PD.
The magnitude of the dose-response association between ACEs and adult life stress did not decrease after adjusting for demographic factors signifying a significant direct relationship between the two. Adult stressors may possibly be mediators of the association between ACEs and PD, demonstrated by the reduction of the effect of ACEs on PD after the addition of adult stress to the model. This means that participants exposed to ACEs may be more likely to show greater distress in the presence of adult stressors compared to those not exposed to ACEs, and that comparatively lower levels of stress may trigger distress [16]. The interaction analysis showed that in individuals that experienced high levels of adult stress, experiencing five ACEs or less, and experiencing six ACEs or more had significantly different effects on PD compared to experiencing no ACEs. Additionally, low ACE levels did 
not have a significant association with PD in the absence of adult stress. High levels of adult stress therefore significantly increased the likelihood of those who had a history of ACEs, especially cumulatively high levels of ACEs, also reporting PD. Although the results also suggest that adult stress on its own has a greater impact on PD than do ACEs, these two exposures possibly interact in several possible ways that have been suggested in previous research.

The interaction between ACEs and adult stressful events is admittedly complex, with ACEs possibly exacerbating the effect of subsequent stress on PD, and adult stress possibly mediating the relationship between ACEs and PD. These pathways are not fully elucidated here but our results are congruent with current knowledge. Evidence shows that exposure to ACEs can trigger neurophysiological sensitivity and erode the stress reactive and adaptive threshold thus stimulating dysfunctional coping strategies [22, 40]. Important psychosocial resources are undermined, resulting in lower levels of perceived social support and poorer perceptions of the self [40]. These shortfalls have negative mediating influences on positive subsequent development and physical, psychological, and social health outcomes in adulthood [40]. In addition to this heightened sensitivity, those who are exposed to ACEs may be at an increased risk of experiencing adult stress. The initial exposure to childhood stressors can lead to secondary effects through the inability to form and sustain healthy relationships, poor academic achievement and making decisions that leave individuals vulnerable to subsequent stressors like those investigated in this study [21]. Individuals exposed to childhood stress are hypothesized to have limited buffering of these secondary stressors compared to individuals without prior significant adversity exposure [21].

An important link between early life and adulthood stress, demonstrated in part through the hierarchical analysis, is through socio-economic status. Individuals exposed to ACEs are more likely to attain lower levels of education which lead to financial insecurity that can increase the risk of personal and family conflict, homelessness and unemployment [5]. Consequent to these adult adversities is the instability of social resources and reduced economic resources available to obtain professional help and maintain healthy habits $[5,41]$.

The Parental Acceptance-Rejection Theory (PARTheory) may be applied to explain the consistent link between neglect and PD in this sample. According to the theory, perceived parental acceptance or rejection affects psychological adjustment in childhood. In addition, when parental rejection that occurred in childhood is recollected later in life, it is likely to be associated with the same form of psychological maladjustment in adulthood [42, 43]. Parental acceptance refers to warmth, affection, love, care, comfort, support and nurturance, while parental rejection refers to the absence or withdrawal of warmth, affection, or love by parents towards their children [44]. An individual's emotional security is partly dependent on the amount of warmth he or she receives from parents as a child and how he or she perceives parents' warmth. Lack of experience of warmth may lead to low self-esteem, negative mental representations of self and others, anger, unresponsiveness, sadness, and emotional instability [45]. Those who report being neglected would be at an increased direct risk of PD in adulthood, apart from the influence of other factors. It is also possible that other forms of adversity such as physical and sexual abuse are perceived by the child as lack of love, comfort and warmth and have the same effect as neglect on the child's and subsequently the adult's psychological well-being.

The GHQ subscales are constructed to each represent common elements of symptomatology [46]. Our analysis therefore sought to assess the association of ACEs with each set of reported symptoms. Three subscales showed results in the expected directions but the social dysfunction subscale was not significantly associated with any of the cumulative ACE variables except high level of ACEs (unadjusted OR 1.16 (95\% CI: 1.01-1.33). The items in the social dysfunction scale may not all be appropriate for our sample. However, women were more likely to report social dysfunction compared to men, those who were married and those who had jobs were less likely to report dysfunction compared to those who were single and unemployed respectively. These socio-demographic factors may have modified the association of ACEs with this subscale.

\section{Strengths and limitations}

Our study utilized data from a long-running cohort with well-established data collection and management methods. Although a birth cohort is not a nationally representative sample two decades later, the data enable us to assess the presence of ACEs in a middle income population and test its association with poor mental well-being in adulthood. The experience of ACEs was reported retrospectively at 22 years of age, which may be subject to recall bias. The role of social coping resources in the association between ACEs and PD has been alluded to previously but we were not able to assess this here [47]. The missing observations may potentially affect the estimations as well but it may either be to under or over estimate.

\section{Conclusion and future implications}

The experience of ACEs in this population compares with that found in other populations and points to the need for a more holistic approach in dealing with childhood adversity that includes identifying other forms of maltreatment and neglect when one adversity is reported. Treatment of mental illness and promotion of mental well-being should not only focus on contemporaneous 
events but also on possible childhood maltreatment and adversity.

Of all animal species, human offspring are dependent on their parents for the longest time. This means that any efforts to support healthy early childhood growth should necessarily include parental support and education. Governments and communities need to work together to support healthy family relationships and support parents in their efforts to raise their children. Effective and collective interventions are necessary in cases of adversity, abuse and violence in the household. Our study shows that this will contribute to improved mental wellbeing and other evidence suggests that it may also prevent other diseases $[4,10]$.

Early childhood development (ECD) is the foundation for sustainable development. Building a strong beginning for healthy development in the early years of life is essential for individual well-being, economic productivity and harmonious societies around the world [38, 48]. Every child, no matter where they live needs the best start in life in order for them to reach their full developmental potential. For the first time in the history of global development, ECD is a major part of the global development goals both directly in lifelong learning and embedded in several other goals. It is therefore time for governments, civil society, businesses, communities and individuals to work together to ensure that the importance of ECD takes center stage and protecting children from adversity is one of the key action points. The current analysis is useful in showing the extent of the problem, albeit in part, and provides a more comprehensive view than studying ACEs as single exposures.

\section{Additional files}

Additional file 1: Questionnaire items used to assess the experience of ACEs. The table presents the questionnaire items derived from the WHO ACE-IQ used to measure the exposure to ACEs. (DOCX $15 \mathrm{~kb}$ )

Additional file 2: Number of participants reporting each of the GHQ subscales stratified by their experience of at least one ACE. The graphs present in each quadrant the participants who reported each of the four GHQ subscales, those who experienced no ACEs on the left of each quadrant and those who experienced at least one ACE on the right. (PDF $177 \mathrm{~kb}$ )

Additional file 3: Separate and cumulative effects of ACEs on adult life stress. The table presents the regression results of ACEs on adult life stress. (DOCX $14 \mathrm{~kb}$ )

Additional file 4: Association between ACEs and the different PD subscales. This table presents the associations between ACEs and the four GHQ subscales, obtained using negative binomial regression. (DOCX $15 \mathrm{~kb}$ )

Additional file 5: Hierarchical regression results: ACEs as binary exposure. Presented here are the adjusted hierarchical regression results using ACE as a binary exposure, including the interaction analysis. (DOCX $14 \mathrm{~kb}$ )

\section{Abbreviations}

ACE: adverse childhood experiences; PD: psychological distress;

SES: socioeconomic status; DALY: disability-adjusted life years; SASH: South African Stress and Health Study; YLD: years lived with disability; Bt20: Birth to Twenty Plus Study; Bt10: Birth to Ten Study; GHQ-28: General Health
Questionnaire 28; DSTV: satellite television; ANOVA: analysis of variance; AOR: adjusted odds ratio; PARTheory: parental acceptance-rejection theory

\section{Acknowledgements}

The support of the DST-NRF Centre of Excellence in Human Development at the University of the Witwatersrand, Johannesburg in the Republic of South Africa towards this research is hereby acknowledged. Opinions expressed and conclusions arrived at, are those of the authors and are not to be attributed to the CoE in Human Development.

\section{Funding}

The Birth to Twenty Plus Study was funded by the SA Medical Research Council, Wellcome Trust (UK) and the University of the Witwatersrand, Johannesburg. MM, SAN and LMR are supported by the DST-NRD Centre of Excellence in Human Development at the University of the Witwatersrand, Johannesburg, South Africa. None of the funders had a role in the design of the study and collection, analysis and interpretation of data, and in writing the manuscript.

\section{Availability of data and materials}

The datasets generated and/or analyzed during the current study are not publicly available due to them containing information that could compromise research participant privacy, but are available from Professor Shane Norris on reasonable request.

\section{Authors' contributions}

All the authors were involved in the conception and design of the study. SAN and LMR were responsible for data acquisition. MM was responsible for data analysis, interpretation of results and drafting the manuscript. All authors read and critically revised the manuscript for important intellectual content and gave their final approval of the version to be published, as well as taking responsibility of all aspects of the work to ensure that all questions related to the work are appropriately resolved.

\section{Ethics approval and consent to participate}

Ethical clearance for this study and the Bt20+ study were obtained from the Human Research Ethics Committee of the University of the Witwatersrand, clearance numbers M160921 and M111182 respectively. All participants were provided with information on the study and its procedures and signed consent forms at enrolment.

\section{Consent for publication}

Not applicable.

\section{Competing interests}

The authors declare that they have no competing interests.

\section{Publisher's Note}

Springer Nature remains neutral with regard to jurisdictional claims in published maps and institutional affiliations.

Received: 21 February 2018 Accepted: 26 June 2018

Published online: 05 July 2018

\section{References}

1. Anda RF, Felitti VJ, Bremner JD, Walker JD, Whitfield C, Perry BD, et al. The enduring effects of abuse and related adverse experiences in childhood. Eur Arch Psychiatry Clin Neurosci. 2006;256(3):174-86.

2. Chapman DP, Whitfield CL, Felitti VJ, Dube SR, Edwards VJ, Anda RF. Adverse childhood experiences and the risk of depressive disorders in adulthood. J Affect Disord. 2004;82(2):217-25.

3. Dube SR, Anda RF, Felitti VJ, Chapman DP, Williamson DF, Giles WH. Childhood abuse, household dysfunction, and the risk of attempted suicide throughout the life span: findings from the adverse childhood experiences study. JAMA. 2001;286(24):3089-96.

4. Edwards VJ, Holden GW, Felitti VJ, Anda RF. Relationship between multiple forms of childhood maltreatment and adult mental health in community respondents: results from the adverse childhood experiences study. Am J Psychiatry. 2003;160(8):1453-60. 
5. Nurius PS, Green S, Logan-Greene P, Borja S. Life course pathways of adverse childhood experiences toward adult psychological well-being: a stress process analysis. Child Abuse Negl. 2015;45:143-53.

6. Schilling EA, Aseltine $\mathrm{RH}$, Gore $\mathrm{S}$. Adverse childhood experiences and mental health in young adults: a longitudinal survey. BMC Public Health. 2007;7(1):30.

7. Jewkes RK, Dunkle K, Nduna M, Jama PN, Puren A. Associations between childhood adversity and depression, substance abuse and HIV and HSV2 incident infections in rural south African youth. Child Abuse Negl. 2010; 34(11):833-41.

8. Centers for Disease Control and Prevention. Adverse childhood experiences reported by adults - five states, 2009. Atlanta, GA, USA: Centers for Disease Control and Prevention; 2010. https://www.cdc.gov/mmwr/pdf/wk/ mm5949.pdf. Accessed 15 June 2017

9. Kathryn Ashton MAB, Alisha R. Davies, Katie Hardcastle and Karen Hughes. Adverse Childhood Experiences and their association with chronic disease and health service use in the Welsh adult population. Cardiff, Wales: Public Health Wales NHS Trust Centre for Public Health, Liverpool John Moores University 2016. http://www.wales.nhs.uk/sitesplus/documents/888/ ACE\%20Chronic\%20Disease\%20report\%20\%289\%29\%20\%282\%29.pdf. Accessed July 2017.

10. Bellis M, Hughes K, Leckenby N, Hardcastle K, Perkins C, Lowey H. Measuring mortality and the burden of adult disease associated with adverse childhood experiences in England: a national survey. J Public Health. 2014;37(3):445-54.

11. Soares ALG, Howe LD, Matijasevich A, Wehrmeister FC, Menezes AM, Gonçalves $\mathrm{H}$. Adverse childhood experiences: prevalence and related factors in adolescents of a Brazilian birth cohort. Child Abuse Negl. 2016;51:21-30.

12. Whiteford HA, Ferrari AJ, Degenhardt L, Feigin V, Vos T. The global burden of mental, neurological and substance use disorders: an analysis from the global burden of disease study 2010. PLoS One. 2015;10(2):1-14.

13. Ferrari AJ, Norman RE, Freedman G, Baxter AJ, Pirkis JE, Harris MG, et al. The burden attributable to mental and substance use disorders as risk factors for suicide: findings from the global burden of disease study 2010. PLoS One. 2014;9(4):e91936.

14. Hay Sl, Abajobir AA, Abate KH, Abbafati C, Abbas KM, Abd-Allah F, et al. Global, regional, and national disability-adjusted life-years (DALYs) for 333 diseases and injuries and healthy life expectancy (HALE) for 195 countries and territories, 1990-2016: a systematic analysis for the global burden of disease study 2016. Lancet. 2017;390(10100):1260-344.

15. Tomlinson M, Grimsrud AT, Stein DJ, Williams DR, Myer L. The epidemiology of major depression in South Africa: results from the South African Stress Health study. SAMJ. 2009;99(5):367-73.

16. Seedat S, Stein DJ, Jackson PB, Heeringa SG, Williams DR, Myer L. Life stress and mental disorders in the south African stress and health study. SAMJ. 2009;99(5):375-82.

17. Promoting Mental WHO. Health: concepts, emerging evidence, practice. Geneva, Switzerland: WHO; 2004. http://www.who.int/mental_health/ evidence/en/promoting_mhh.pdf. Accessed 6 Sept 2017

18. Drapeau A, Marchand A, Beaulieu-Prévost D. Epidemiology of psychological distress. In: L' Abate L, editor. Mental illnesses-understanding, prediction and control. Rijeka, Croatia: InTech; 2012. p. 105-34. https://www.intechopen. com/books/mental-illnesses-understanding-prediction-and-control/ epidemiology-of-psychological-distress. Accessed 13 Feb 2018.

19. Payton AR. Mental health, mental illness, and psychological distress: same continuum or distinct phenomena? J Health Soc Behav. 2009;50(2):213-27.

20. Jackson PB, Williams DR, Stein DJ, Herman A, Williams SL, Redmond DL. Race and psychological distress: the south African stress and health study. J Health Soc Behav. 2010;51(4):458-77.

21. Nurius PS, Uehara E, Zatzick DF. Intersection of stress, social disadvantage, and life course processes: reframing trauma and mental health. Am J Psychiatr Rehabil. 2013;16(2):91-114.

22. Pearlin LI, Schieman S, Fazio EM, Meersman SC. Stress, health, and the life course: some conceptual perspectives. J Health Soc Behav. 2005;46(2):205-19.

23. Peltzer K, Pengpid S, Skaal L. Prevalence of psychological distress and associated factors in urban hospital outpatients in South Africa. S Afr J Psych. 2012;18(1):10-5

24. Erdem Ö, Van Lenthe FJ, Prins RG, Voorham TA, Burdorf A. Socioeconomic inequalities in psychological distress among urban adults: the moderating role of neighborhood social cohesion. PLoS One. 2016;11(6):e0157119.

25. Pigeon-Gagné É, Hassan G, Yaogo M, Ridde V. An exploratory study assessing psychological distress of indigents in Burkina Faso: a step forward in understanding mental health needs in West Africa. Int J Equity Health. 2017;16:1-11.
26. Kessler RC, McLaughlin KA, Green JG, Gruber MJ, Sampson NA, Zaslavsky AM, et al. Childhood adversities and adult psychopathology in the WHO world mental health surveys. Br J Psychiatry. 2010;197(5):378-85.

27. Richter L, Norris S, Pettifor J, Yach D, Cameron N. Cohort Profile: Mandela's children: The 1990 birth to twenty study in South Africa. Int J Epidemiol. 2007;36(3):504-11.

28. Population of 2017. POPULATION OF SOWETO 2017. 2017. http:// populationof2017.com/population-of-soweto-2017.html. Accessed 18 Jan 2018.

29. Kagura J, Adair LS, Musa MG, Pettifor JM, Norris SA. Blood pressure tracking in urban black south African children: birth to twenty cohort. BMC Pediatr. 2015;15(1):1.

30. Sterling M. General health questionnaire-28 (GHQ-28). J Physiother. 2011; 57(4):259.

31. Dube SR, Felitti VJ, Dong M, Giles WH, Anda RF. The impact of adverse childhood experiences on health problems: evidence from four birth cohorts dating back to 1900. Prev Med. 2003;37(3):268-77.

32. Finkelhor D, Shattuck A, Turner $H$, Hamby S. A revised inventory of adverse childhood experiences. Child Abuse Negl. 2015;48:13-21.

33. Sheppard ZA, Norris SA, Pettifor JM, Cameron N, Griffiths PL. Approaches for assessing the role of household socioeconomic status on child anthropometric measures in urban South Africa. Am J Hum Biol. 2009;21(1):48-54.

34. Merrick MT, Ports KA, Ford DC, Afifi TO, Gershoff ET, Grogan-Kaylor A. Unpacking the impact of adverse childhood experiences on adult mental health. Child Abuse Negl. 2017:69:10-9.

35. Caron J, Fleury M-J, Perreault M, Crocker A, Tremblay J, Tousignant M, et al. Prevalence of psychological distress and mental disorders, and use of mental health services in the epidemiological catchment area of Montreal south-west. BMC Psychiatry. 2012;12(1):183.

36. Cluver L, Orkin M, Boyes ME, Sherr L. Child and adolescent suicide attempts, suicidal behavior, and adverse childhood experiences in South Africa: a prospective study. J Adolesc Health. 2015;57(1):52-9.

37. Raposa EB, Hammen CL, Brennan PA, O'callaghan F, Najman JM. Early adversity and health outcomes in young adulthood: the role of ongoing stress. Health Psychol. 2014;33(5):410.

38. Shonkoff JP, Richter L, van der Gaag J, Bhutta ZA. An integrated scientific framework for child survival and early childhood development. Pediatrics. 2012;129(2):e460-e72.

39. Shonkoff JP. Building a new biodevelopmental framework to guide the future of early childhood policy. Child Dev. 2010;81(1):357-67.

40. Nurius PS, Green S, Logan-Greene P, Longhi D, Song C. Stress pathways to health inequalities: embedding ACEs within social and behavioral contexts. Int J Public Health. 2016:8(2):241.

41. Hobfoll SE. The influence of culture, community, and the nested-self in the stress process: advancing conservation of resources theory. Appl Psychol. 2001;50(3):337-421.

42. Rohner RP, Khaleque A. Testing central postulates of parental acceptancerejection theory (PARTheory): a meta-analysis of cross-cultural studies. J Fam Theory Rev. 2010;2(1):73-87.

43. Rohner RP, Khaleque A, Cournoyer DE. Parental acceptance-rejection: theory, methods, cross-cultural evidence, and implications. Ethos. 2005;33(3):299-334.

44. Khaleque A. Perceived parental warmth, and Children's psychological adjustment, and personality dispositions: a meta-analysis. J Child Fam Stud. 2013;22(2):297-306

45. Sart ZH, Börkan B, Erkman F, Serbest S. Resilience as a mediator between parental acceptance-rejection and depressive symptoms among university students in Turkey. J Couns Dev. 2016;94(2):195-209.

46. Molina J, Andrade-Rosa C, González-Parra S, Blasco-Fontecilla H, Real M, Pintor $C$. The factor structure of the general health questionnaire $(\mathrm{GHQ})$ : a scaled version for general practice in Spain. Eur Psychiatry. 2006;21(7):478-86.

47. Cheong EV, Sinnott C, Dahly D, Kearney PM. Adverse childhood experiences (ACEs) and later-life depression: perceived social support as a potential protective factor. BMJ Open. 2017;7(9):e013228.

48. ARNEC. Asia Pacific Regional Network for Early Chldhood. ECD in the SDGs: Abriefing Note. Singapore: ARNEC; 2016. http://www.itacec.org/ece/ document/learning_resources/2017/ECD-in-the-SDGs-20Mar2016_final-002. pdf. 18 Jan 2018. 